\title{
RESPONSE OF VALENCIA ORANGE TREES TO SOME SOURCES OF ORGANIC FERTILIZERS UNDER DIFFERENT TYPES OF SOIL
}

\author{
Ensherah A.H. Tayeh*; M.A. Zaghloul**; M.A. Abou- Seeda*** \\ and A.S. Taalab**** \\ *Hort. Res. Instit., Agric. Res. Center, Giza, Egypt \\ **Soils and Water use Dept. National Res. Center, Dokki, Giza. \\ ***Plant Nutrition Dept. National Res. Center, Dokki, Giza.
}

\section{ABSTRACT}

The investigation was carried out during two successive seasons 2008 and 2009 on Valencia orange trees twenty years old, budded on sour orange rootstock at three types of soil i.e, silty loam of a private citrus orchard of Belbais, Sharkia governorate, loamy sand of a private farm of Abshway, Fayoum governorate and sandy soil at El- Horia farm at ElNobaria sector, El-Behera governorate to study the effect of different sources of organic manure fertilizers i.e. (poultry manure, sheep manure, cattle manure, sewage sludge compost and town waste compost at the rate of $71.4 \mathrm{~kg} /$ tree, Rock phosphate $\left(24.5 \% \mathrm{P}_{2} \mathrm{O}_{5}\right)$ was the source of phosphate fertilizer and Felspar $\left(7.9 \% \mathrm{~K}_{2} \mathrm{O}\right)$ was the source of potassium on comparing with mineral fertilizer [ $800 \mathrm{gm}$ ammonium sulphate $20.5 \% \mathrm{~N}$, $400 \mathrm{gm}$ Calcium super phosphate $15.5 \% \mathrm{P}_{2} \mathrm{O}_{5}$ and $400 \mathrm{gm}$ Potassium sulphate $48 \% \mathrm{~K}_{2} \mathrm{O}$ ] per tree on vegetative growth, leaf (water, pigment and mineral) contents, fruiting parameters (fruit set, June drop and fruiting) percentage, yield and fruit quality. In addition the amounts of $\mathrm{N}, \mathrm{NH}_{4}$, $\mathrm{NO}_{3}, \mathrm{P}, \mathrm{K}, \mathrm{Fe}, \mathrm{Mn}, \mathrm{Zn}$ and $\mathrm{Cu}$ that remained in each studied soil at the end of the experiment were also studied. The obtained results revealed that vegetative growth, leaf water, chlorophylls and mineral contents, fruit set, fruiting, yield and fruit quality significantly increased as a result of organic manure addition specially when using poultry manure, whereas, leaf carotene and June drop \%were lowered. Silty loam soil was the best on affecting trees growth followed by loamy sand whereas sandy soil was the lowest. Adding poultry manure led to an increase in soil $\mathrm{N}, \mathrm{NH}_{4}$ and $\mathrm{P}$, whereas, using chemical fertilizers led to an increase in soil $\mathrm{NO}_{3}$. Applying cattle manure led to an increase in soil $\mathrm{K}$ and Fe. The main observation was that using Town waste compost led to an increase in soil $\mathrm{Zn}$ and $\mathrm{Cu}$.

Key words: Valencia, orange trees, sources of organic fertilizers, different types of soil

\section{INTRODUCTION}

Organic manure can play an important role in modern agriculture. The application of these materials is an important aspect to sustain soil productivity and to maintain beneficial soil biological, chemical and physical properties (Abou Seeda, 1987). Egyptian soils are low in organic matter about $2 \%$ Balba, (1976). Now with increasing the cost of mineral fertilizers there is renewed interest in organic recycling to improve soil fertility and productivity. So, organic wastes compost may be utilized in soil as source of nutrients for production Warr and Hormick, (1990). Poultry manure was used as organic fertilizer. Unemya and Sekiya, (1985) pointed out that

Fayoum J. Agric. Res. \& Dev., Vol.25, No.2, July, 2011 
$\mathrm{N}, \mathrm{P}, \mathrm{K}, \mathrm{Ca}$ and $\mathrm{Mg}$ of soil increase by the application of Poultry manure. Organic matter improve the nutritional status of most soils, In particular sandy soil. El- Aila et. al.,(2001). Fliessbach et. al., (2000). found that organic manure application increased the transfer elements between the solid phase and soil solution, They also reported that organic soil management improved the soil structure by increasing soil activity, thus reducing the risk of soil erosion and promoted the development of earth worms and aboveground arthropods. This action can improve the growing condition for the trees.

Concerning the effect of organic manure, Abou- Sayed, (1997). on Balady mandarin stated that trees fertilized with compost chicken manure showed significant increase in growth, Abdel-Nasser and Harhash(2000) found that organic manure had positively affect on soil water- holdin capacity which led to increase leaf water contents. Chokha, et. al., (1993). on sweet orange trees mentioned that using organic manure gave satisfactory increase in yield and fruit quality of the trees. ElKobbiaobbia, (1999). on Navel orange trees stated that flowering parameters were promoted in the presence of organic fertilizer. Grassi, et. al., (1999). On Rangpour Lime obtained a significant increment in yield and fruit quality due to adding organic manure. Motskobili, (1984). on Satsuma mandarin observed that applying manure significantly increased shoot length, leaves number per shoot ,leaf area and yield. Huang, et. al., (1995). on Satsuma mandarin trees reported that all organic fertilizers treatments produced the highest number of fruits as well as the highest yield, contained significant increment in N-P contents comparing with NPK fertilizers. Helail, et. al., (2003). on Washington navel orange trees in response to organic manure as compared to mineral fertilizer treatments suggested that, under organic system fruit of citrus trees had more Vitamin C. and increased the amount of total sugars. Thus, the aim of this research was to study the effect of adding various organic manure fertilizers compared with mineral fertilizers on vegetative growth, leaf water content, leaf pigment and mineral contents, fruit set\%, June drop\%, fruiting $\%$, yield and fruit quality besides, the residue of total nitrogen, $\mathrm{NH}_{4}$ and $\mathrm{NO}_{3}$ in soil . In addition studying the effect of organic manure in improving the available soil nutrients i.e. $\mathrm{P}, \mathrm{K}, \mathrm{Fe}, \mathrm{Zn}, \mathrm{Mn}$ and $\mathrm{Cu}$ at the end of the experiment was also achieved.

\section{MATERIALS AND METHODS}

The investigation was carried out during two successive seasons 2008 and 2009 on Valencia orange trees twenty years old, budded on sour orange rootstock at three types of soil i.e, silty loam of a private citrus orchard of Belbais, Sharkia governorate, loamy sand of a private farm of Abshway, Fayoum Governorate and sandy soil at El- Horia farm at El-Nobaria sector, El-Behera governorate to study the effect of different sources of organic manure fertilizers i.e. (poultry manure, sheep manure, cattle manure, sewage sludge compost and town waste compost to comparing with mineral fertilizer on vegetative growth, leaf (water, pigment and mineral) contents, fruiting parameters (fruit set, June drop and fruiting) percentage, yield and fruit quality. In addition to study the amount of $\mathrm{N}, \mathrm{NH}_{4}, \mathrm{NO}_{3}, \mathrm{P}, \mathrm{K}, \mathrm{Fe}, \mathrm{Mn}$, $\mathrm{Zn}$ and $\mathrm{Cu}$ that remained in each studied soil at the end of the experiment. Trees were planted at $5 \times 5$ meters apart, nearly similar in their growth as possible. The treatments of the experiment were arranged in a complete randomized block design and each treatment was replicated three times with three trees per each replicate. NPK

Fayoum J. Agric. Res. \& Dev., Vol.25, No.2, July, 2011 
RESPONSE OF VALENCIA ORANGE TREES TO..........

chemical fertilizer [800gm ammonium sulphate $20.5 \% \mathrm{~N}, 400 \mathrm{gm}$ Calcium super phosphate $15.5 \% \mathrm{P}_{2} \mathrm{O}_{5}$ and $400 \mathrm{gm}$ Potassium sulphate $48 \% \mathrm{~K}_{2} \mathrm{O}$ ] per tree was applied as control. The amount of ammonium sulphate was divided into three doses and added each dose to the soil in January, March and August. While, calcium super phosphate added as one dose in January, whereas, potassium sulphate was divided into two equal doses and added in March and August. Anyhow, the amount of organic manure fertilizer was applied at the rate of 12 Tons per feddan (168 trees) i.e., $71.4 \mathrm{~kg} /$ tree. Organic manure was soaked in 30 liter of water for $24 \mathrm{hrs}$. before using and mixed with the surface of soil layer $(0-20) \mathrm{cm}$. All organic manure were divided into three doses and added in January, March and August. Organic fertilizers were analyzed and presented in Table (1). Rock phosphate $\left(24.5 \% \mathrm{P}_{2} \mathrm{O}_{5}\right)$ was the source of phosphate fertilizer and was added at the rate of $200 \mathrm{gm} /$ tree at January, Felspar $\left(7.9 \% \mathrm{~K}_{2} \mathrm{O}\right)$ was the source of potassium which divided into two equal batches and was applied at March and August.

Table (1): Chemical analysis of organic manures used during the experiment.

\begin{tabular}{|c|c|c|c|c|c|c|c|c|}
\hline $\begin{array}{c}\text { Organic } \\
\text { manure }\end{array}$ & $\begin{array}{c}\text { Organic } \\
\text { matter }(\boldsymbol{\%})\end{array}$ & $\begin{array}{c}\mathbf{N} \\
(\boldsymbol{\%})\end{array}$ & $\begin{array}{c}\mathbf{P} \\
(\boldsymbol{\%})\end{array}$ & $\begin{array}{c}\mathbf{K} \\
(\mathbf{\%})\end{array}$ & $\begin{array}{c}\mathbf{F e} \\
(\mathbf{\%})\end{array}$ & $\begin{array}{c}\mathbf{M n} \\
(\mathbf{P P m})\end{array}$ & $\begin{array}{c}\mathbf{Z n} \\
(\mathbf{P P m})\end{array}$ & $\begin{array}{c}\mathbf{C u} \\
(\mathbf{P P m})\end{array}$ \\
\hline Poultry manure & 73.2 & 2.53 & 1.35 & 0.75 & 0.84 & 196 & 174 & 125 \\
\hline Sheep manure & 65.4 & 1.99 & 1.20 & 0.61 & 1.21 & 187 & 200 & 100 \\
\hline Cattle manure & 60.7 & 1.01 & 0.80 & 0.70 & 1.33 & 264 & 98 & 87 \\
\hline Sewage sludge & 45.6 & 1.63 & 1.09 & 0.38 & 1.09 & 219 & 224 & 239 \\
\hline Town Waste & 42.3 & 0.85 & 0.69 & 0.42 & 0.92 & 180 & 163 & 140 \\
\hline
\end{tabular}

The trees had received nearly the same other management practices.

Studied parameters:-

1-Vegetative growth: Shoot length $(\mathrm{cm})$, leaves number per shoot and leaf area $(\mathrm{cm} 2)$ according to Watson,(1985).

2-Leaf analysis:

a-Leaf water contents was determined according to Weatherly (1950) method.

b-Leaf pigments: Chlorophyll(a\&b) and carotene contents were determined using the method described by Holden(1965).

c-Leaf mineral contents: Leaf $\mathrm{N}$ content using the method described by Pregl (1945), d-Leaf P content using the method described by Jackson(1958).

e-Leaf K content using the method described by Brown \& Lilleland (1946).

f-Leaf Fe,Zn and Mn content using the method described by Capman and Pratt(1961).

3-Fruiting parameters: Fruit set $\%$, fruit drop $\%$ and fruiting $\%$ calculated according to the equations given by Vyvyan (1946).

4-- yield: At harvesting time (mid April) fruits of each treatment were harvested then yield was recorded including number of fruits per tree and fruit weight (gm) were recorded, estimated yield as $\mathrm{kg} / \mathrm{tree}$, Tons per feddan was also concerned as yield $\mathrm{kg} /$ treex 168 tree in feddan.

5- Fruit quality: Ten fruits were sampled from each tree to determine certain fruit characteristics as follow: Fruit weight $(\mathrm{gm})$, fruit size $\left(\mathrm{cm}^{3}\right)$ and juice percentage, Total soluble solids (T.S.S.\%) in fruit juice using a hand refractometer Abbe, Total acidity\% and Ascorbic acid (mg/100ml juice) content were determined according to A.O.A.C.(1975). In addition, T.S.S./ acid ratio was also calculated.

Fayoum J. Agric. Res. \& Dev., Vol.25, No.2, July, 2011 
6- Soil analysis: Soils were sampled at the depth of $60 \mathrm{~cm}$ of the three studied soils to determine total $\mathrm{N}, \mathrm{NO} 3, \mathrm{NH}_{4}, \mathrm{P}, \mathrm{K}, \mathrm{Fe}, \mathrm{Zn}, \mathrm{Mn}$ and $\mathrm{Cu}$ according to Jackson (1958) and Wilde et. al.,(1985). at the starting (Table2) and at the end of the experiment (Tables11\&12).

7- Statistical analysis: The obtained data of each season were statistically analyzed using the procedure outlined by Snedecor and Cochran (1985). Mean separation

Table (2) Physical and Chemical analysis of the three types of soil.

\begin{tabular}{|c|c|c|c|c|c|c|c|}
\hline \multirow{2}{*}{$\begin{array}{l}\text { Soil } \\
\text { texture }\end{array}$} & \multirow{2}{*}{$\begin{array}{c}\text { E.C. } \\
\mathrm{ms} / \mathrm{cm}\end{array}$} & \multirow[t]{2}{*}{$\mathrm{pH}$} & \multicolumn{5}{|c|}{ Mechanical analysis } \\
\hline & & & Sand $\%$ & Silt\% & Clay\% & O.M.\% & $\mathrm{CaCo}_{3} \%$ \\
\hline Silty loam & 0.62 & 8.45 & 7.3 & 72.59 & 17.7 & 1.86 & 0.55 \\
\hline Loamy sand & 0.92 & 8.01 & 61.46 & 23.0 & 8.8 & 0.80 & 5.94 \\
\hline \multirow[t]{4}{*}{ Sandy } & 1.7 & 7.85 & 86.56 & 2.6 & 3.1 & 0.04 & 7.70 \\
\hline & & \multicolumn{6}{|c|}{ Chemical analysis } \\
\hline & & \multicolumn{6}{|c|}{$\mathrm{mg} / \mathrm{kg}$ soil } \\
\hline & & \multicolumn{2}{|c|}{ Total N } & \multicolumn{2}{|c|}{$\mathrm{NO}_{3}$} & \multicolumn{2}{|c|}{$\mathrm{NH}_{4}$} \\
\hline \multicolumn{2}{|l|}{ Silty loam } & \multicolumn{2}{|c|}{39.9} & \multicolumn{2}{|c|}{14.9} & \multicolumn{2}{|c|}{8.10} \\
\hline Loamy sanc & & & .1 & \multirow{2}{*}{\multicolumn{2}{|c|}{$\frac{6.90}{3.30}$}} & \multicolumn{2}{|c|}{3.4} \\
\hline \multirow{3}{*}{\multicolumn{2}{|c|}{ Sandy }} & \multicolumn{2}{|c|}{$\frac{19.1}{7.1}$} & & & & 70 \\
\hline & & \multicolumn{6}{|c|}{ Available soil nutrients, $\mathrm{mg} / \mathrm{kg}$ soil } \\
\hline & & $\mathrm{P}$ & $\mathrm{K}$ & $\mathrm{Fe}$ & $\mathrm{Zn}$ & $\mathrm{Mn}$ & $\mathrm{Cu}$ \\
\hline \multicolumn{2}{|l|}{ Silty loam } & 21.4 & 22.5 & 2.4 & 0.8 & 1.9 & 0.33 \\
\hline \multicolumn{2}{|l|}{ Loamy sand } & 18.3 & 23.2 & 3.8 & 1.6 & 2.9 & 1.1 \\
\hline \multicolumn{2}{|l|}{ Sandy } & 5.6 & 16.19 & 1.14 & 0.41 & 0.16 & 0.8 \\
\hline
\end{tabular}

\section{RESULTS AND DISCUSSION}

\section{Growth parameters and leaf water content (\%):}

It is clear from Table (3) that supplying Valencia orange trees with all organic sources was significantly improved shoots length $(\mathrm{cm})$, number of leaves per shoot, leaf area $\left(\mathrm{cm}^{2}\right)$ and leaf water content $(\%)$, results indicated that organic fertilizers were more effective in increasing vegetative growth and leaf water content than mineral ones in all types of soil due to improving water- holding capacity of soil. The highest values were obtained by adding poultry manure. On the other hand, applying sewage sludge and town waste composts had little vegetative growth and leaf water content than control. The lowest value of growth was obtained by adding town waste due to the poor contents of macro and micro nutrients. Vegetative growth characters increased by using poultry manure could be interpreted that it contains twice amount of nitrogen and much $\mathrm{P}$ and $\mathrm{K}$ than others.

Similar results were reported by many investigators such as Chokha, et. al., (1993). on sweet orange trees mentioned that using organic manure gave satisfactory increase in vegetative growth and leaf water contents. Abdel-Nasser and Harhash (2000) found that organic manure had positively affect on soil water- holdin capacity which led to increase leaf water contents.

Fayoum J. Agric. Res. \& Dev., Vol.25, No.2, July, 2011 
Table 3

Fayoum J. Agric. Res. \& Dev., Vol.25, No.2, July, 2011 
Leaf pigment and mineral contents:

Leaf chlorophyll (a \& b) contents, Table (4) respond statistically by the different sources of organic fertilizers and recorded an increase while, decreased leaf carotene content. It is quite evident from Table (5) that leaf $\mathrm{N}, \mathrm{P}, \mathrm{K}$ content were significantly affected by adding organic fertilizers. The results also indicated the improving effect of organic sources on leaf N,P and K. It could be arranged in the following descending order, $\mathrm{N}$ (poultry manure, cattle manure\& sewage sludge, sheep manure, mineral fertilizer (control) \& town waste. P (cattle manure, sewage sludge, poultry manure, sheep manure town waste, mineral fertilizer (control). It is clear from data of Table (6) that leaf Fe content was the largest when cattle manure source was applied which occupied the first rank, then sewage sludge compost, poultry manure, sheep manure and town waste in descending order. While, control had the lowest values. Leaf $\mathrm{Zn}$ content as a results of treated organic manure fertilizers were the largest when sewage sludge compost was applied, followed by sheep manure, town waste, poultry manure, cattle manure, town waste compost, control in descending order. Leaf $\mathrm{Mn}$ on the other hand, affected with source of organic compost and had the largest content by fertilizing with cattle manure, whilst, the lowest result was achieved by using town waste. These results were true in the three soil experiments. The values of these parameters were greater in silty loam, sandy loam and sandy soil in descending order. The same conclusion was observed by Huang et. al, (1995) and Abou- Sayed (1997).

Table (4): Leaf chlorophyll (a \& b) and carotene ( $\mathrm{mg} / 100 \mathrm{gm}$ fresh weight of Valencia orange trees as affected by different sources of organic fertilizer at different soil types.

\begin{tabular}{|c|c|c|c|c|c|c|c|c|c|c|}
\hline \multicolumn{2}{|c|}{ Leaf pigment contents } & \multicolumn{3}{|c|}{ Chlorophyll(a) } & \multicolumn{3}{|c|}{ Chlorophyll(b) } & \multicolumn{3}{|c|}{ Carotene } \\
\hline Soil type (A) & Fertilizer source (B) & 2008 & 2009 & mean & 2008 & 2009 & mean & 2008 & 2009 & mean \\
\hline \multirow[t]{7}{*}{ Silty loamy } & Poultry manure & 143.7 & 145.9 & 144.80 & 76.1 & 76.2 & 76.15 & 26.3 & 26.2 & 26.25 \\
\hline & Sheep manure & 125.0 & 127.1 & 126.05 & 68.9 & 69.1 & 69.00 & 27.6 & 27.4 & 27.50 \\
\hline & Cattle manure & 138.8 & 140.0 & 139.40 & 79.7 & 81.9 & $\mathbf{8 0 . 8 0}$ & 27.4 & 27.2 & 27.30 \\
\hline & Sewage sludge & 118.6 & 119.7 & 119.15 & 50.9 & 51.1 & 51.00 & 39.7 & 39.5 & 39.60 \\
\hline & Town waste & 115.0 & 116.1 & 115.55 & 47.3 & 47.5 & 47.40 & 36.6 & 36.4 & 36.50 \\
\hline & Control(NPK) & 123.3 & 123.9 & 123.60 & \begin{tabular}{|l|}
64.9 \\
\end{tabular} & 65.0 & 64.95 & 28.6 & 28.4 & 28.50 \\
\hline & Mean & 127.4 & 128.63 & 128.01 & 64.53 & 65.13 & 64.88 & 31.03 & 30.85 & 30.94 \\
\hline \multirow[t]{7}{*}{ Loamy sand } & Poultry manure & 139.0 & 141.0 & 140.0 & \begin{tabular}{|l|}
72.8 \\
\end{tabular} & 72.9 & 72.85 & 26.5 & 26.3 & 26.40 \\
\hline & Sheep manure & 119.8 & 121.0 & 120.40 & 67.1 & 67.4 & 67.25 & 27.8 & 27.6 & 27.70 \\
\hline & Cattle manure & 133.7 & 130.8 & 134.25 & 68.9 & 69.1 & 69.00 & 27.7 & 27.4 & 27.55 \\
\hline & Sewage sludge & 113.0 & 113.9 & 113.45 & 47.4 & 47.9 & 47.65 & 40.4 & 40.3 & 40.35 \\
\hline & Town waste & 109.4 & 110.3 & 109.85 & 43.8 & 43.9 & 43.85 & 37.3 & 37.2 & 37.25 \\
\hline & Control(NPK) & 117.8 & 118.9 & 118.35 & 61.9 & 62.1 & 62.0 & 29.5 & 29.2 & 29.35 \\
\hline & $\begin{array}{c}\text { Mean } \\
\end{array}$ & 122.12 & 123.32 & 122.72 & 60.32 & 60.88 & 60.60 & 31.53 & 31.33 & 31.43 \\
\hline \multirow[t]{7}{*}{ Sandy } & Poultry manure & 128.6 & 129.6 & 129.10 & 67.6 & 68.7 & 68.15 & 27.2 & 23.9 & 25.55 \\
\hline & Sheep manure & 108.7 & 109.6 & 109.15 & 64.8 & 65.1 & 64.95 & 28.3 & 27.8 & 28.05 \\
\hline & Cattle manure & 123.6 & 124.5 & 124.05 & 66.2 & 67.1 & 66.65 & 28.2 & 27.6 & 27.90 \\
\hline & Sewage sludge & 102.2 & 103.2 & 102.70 & 45.2 & 46.1 & 45.65 & 42.6 & 41.3 & 41.95 \\
\hline & Town waste & 98.0 & 99.1 & 98.55 & 41.0 & 42.2 & 41.60 & 39.5 & 38.2 & 38.85 \\
\hline & Control(NPK) & 107.6 & 108.7 & 108.15 & 59.6 & 60.1 & $\mathbf{5 9 . 8 5}$ & 30.7 & 30.5 & 30.60 \\
\hline & Mean & 111.45 & 112.45 & 111.95 & 57.4 & 58.22 & $\mathbf{5 7 . 8 0}$ & 32.75 & 31.55 & 32.15 \\
\hline \multirow[t]{2}{*}{ L.S.D. at 5\% } & Soil type (A) & 0.114 & 0.112 & & $\mathbf{0 . 0 3}$ & 0.06 & & 0.004 & 0.003 & \\
\hline & Fertilizer source $(\mathrm{B})$ & 0.235 & 0.249 & & 0.26 & 0.28 & & 0.015 & 0.019 & \\
\hline Interaction & $(\mathrm{A}) \mathbf{X}(\mathrm{B})$ & 0.671 & 0.731 & & 0.45 & 0.58 & & 0.111 & 0.117 & \\
\hline
\end{tabular}

Fayoum J. Agric. Res. \& Dev., Vol.25, No.2, July, 2011 
Table (5): Leaf N,P and $K(\%)$ contents of Valencia orange trees as affected by different sources of organic fertilizer at different soil types.

\begin{tabular}{|c|c|c|c|c|c|c|c|c|c|c|}
\hline \multicolumn{2}{|c|}{ Leaf NPK contents $(\%)$} & \multicolumn{3}{|c|}{$\mathbf{N}$} & \multicolumn{3}{|c|}{$\mathbf{P}$} & \multicolumn{3}{|c|}{$\mathbf{K}$} \\
\hline Soil type (A) & $\begin{array}{c}\text { Fertilizer source } \\
\text { (B) }\end{array}$ & 2008 & 2009 & mean & 2008 & 2009 & mean & 2008 & 2009 & mean \\
\hline \multirow[t]{7}{*}{ Silty loamy } & Poultry manure & 2.90 & 3.00 & 2.95 & \begin{tabular}{|l}
0.62 \\
\end{tabular} & 0.64 & 0.63 & 1.96 & 1.75 & 1.85 \\
\hline & Sheep manure & 2.70 & 2.80 & 2.75 & 0.55 & 0.57 & 0.56 & 1.82 & 1.62 & 1.72 \\
\hline & Cattle manure & 2.80 & 2.90 & 2.85 & 0.74 & 0.76 & 0.75 & 1.71 & 1.42 & 1.56 \\
\hline & Sewage sludge & 2.80 & 2.90 & 2.85 & 0.63 & 0.66 & 0.65 & 1.75 & 1.41 & 1.58 \\
\hline & Town waste & 2.60 & 2.70 & 2.65 & 0.52 & 0.53 & 0.52 & 1.68 & 1.34 & 1.51 \\
\hline & Control(NPK) & 2.61 & 2.66 & 2.63 & 0.42 & 0.44 & 0.43 & 1.60 & 1.48 & 1.54 \\
\hline & Mean & 2.74 & 2.83 & 2.78 & 0.58 & 0.60 & 0.59 & 1.75 & 1.50 & 1.63 \\
\hline \multirow[t]{7}{*}{ Loamy sand } & \begin{tabular}{|l|} 
Poultry manure \\
\end{tabular} & 2.60 & 2.70 & 2.65 & 0.49 & 0.51 & 0.50 & 1.74 & 1.49 & 1.61 \\
\hline & Sheep manure & 2.40 & 2.50 & 2.45 & 0.32 & 0.34 & 0.33 & 1.52 & 1.38 & 1.45 \\
\hline & Cattle manure & 2.50 & 2.60 & 2.55 & 0.52 & 0.54 & 0.53 & 1.41 & 1.26 & 1.34 \\
\hline & Sewage sludge & 2.50 & 2.55 & 2.52 & 0.50 & 0.51 & 0.50 & $\mathbf{1 . 3 5}$ & 1.25 & 1.30 \\
\hline & Town waste & 2.30 & 2.40 & 2.35 & 0.39 & 0.41 & 0.40 & 1.28 & 1.18 & 1.23 \\
\hline & Control(NPK) & 2.40 & 2.43 & 2.42 & 0.28 & 0.29 & 0.29 & 1.38 & 1.28 & 1.33 \\
\hline & \begin{tabular}{|c|} 
Mean \\
\end{tabular} & 2.45 & 2.53 & 2.49 & 0.42 & 0.43 & 0.43 & 1.45 & 1.31 & 1.38 \\
\hline \multirow[t]{7}{*}{ Sandy } & Poultry manure & 2.50 & 2.60 & 2.55 & 0.52 & 0.53 & 0.53 & 1.44 & 1.32 & 1.38 \\
\hline & Sheep manure & 2.30 & 2.40 & 2.35 & 0.34 & 0.36 & 0.35 & 1.22 & 1.18 & 1.20 \\
\hline & Cattle manure & 2.40 & 2.50 & 2.45 & 0.56 & 0.58 & 0.57 & 1.21 & 1.15 & 1.18 \\
\hline & Sewage sludge & 2.43 & 2.59 & 2.52 & 0.53 & 0.54 & 0.54 & 1.19 & 1.15 & 1.17 \\
\hline & Town waste & 2.21 & 2.30 & 2.25 & 0.42 & 0.46 & 0.43 & 1.12 & 1.08 & 1.10 \\
\hline & Control(NPK) & 2.32 & 2.35 & 2.33 & 0.29 & 0.31 & 0.30 & 1.90 & 1.80 & 1.85 \\
\hline & Mean & 2.36 & 2.46 & 2.41 & 0.44 & 0.46 & 0.45 & 1.35 & 1.28 & 1.31 \\
\hline \multirow[t]{2}{*}{ L.S.D. at 5\% } & Soil type (A) & 0.22 & 0.29 & & $\mathbf{0 . 0 7}$ & 0.09 & & 0.08 & $\mathbf{0 . 0 7}$ & \\
\hline & Fertilizer source (B) & 0.34 & 0.53 & & 0.10 & 0.13 & & 0.11 & 0.13 & \\
\hline Interaction & $(\mathbf{A}) \mathbf{X}(\mathbf{B})$ & 0.81 & 0.88 & & 0.22 & 0.24 & & 0.27 & 0.32 & \\
\hline
\end{tabular}

Fruiting :

1-Fruit set \%:

It is clear from Table (7) that Valencia orange trees had the highest fruit set\% when fertilized with poultry manure while, town waste had the lowest affect, these results were recorded in all soil types. Silty loam soil had the best percentage in this concern.

\section{2-June drop\%:}

Table (7) on the other hand, revealed that June drop\% increased by adding town waste fertilizer in sandy soil while, poultry manure fertilizer had the lowest fruit June drop\% in silty loam soil.

\section{3-Fruiting\%:}

The same Table (7) cleared that trees planted in silty loam soil and fertilized with poultry manure fertilizer had the largest fruiting percentage while, the opposite was true in sandy soil especially when adding town waste or sewage sludge fertilizer.

These results are confirmed by the findings of Chokha, et. al., (1993). on sweet orange trees, Abou- Sayed, (1997). on Balady mandarin and El- Kobbiaobbia, (1999). on Navel orange trees mentioned that using organic manure gave satisfactory increase in fruit set $\%$ and fruiting and decrease June drop\% of the trees.

Fayoum J. Agric. Res. \& Dev., Vol.25, No.2, July, 2011 
Table (6): Leaf Fe, Zn and Mn (ppm) contents of Valencia orange trees as affected by different sources of organic fertilizer at different soil types.

\begin{tabular}{|c|c|c|c|c|c|c|c|c|c|c|}
\hline \multicolumn{2}{|c|}{ Leaf $\mathrm{Fe}, \mathrm{Zn}$ and $\mathrm{Mn}(\mathrm{ppm})$} & \multicolumn{3}{|c|}{$\mathbf{F e}$} & \multicolumn{3}{|c|}{$\mathbf{Z n}$} & \multicolumn{3}{|c|}{ Mn } \\
\hline Soil type (A) & Fertilizer source (B) & 2008 & 2009 & mean & 2008 & 2009 & mean & 2008 & 2009 & mean \\
\hline \multirow[t]{7}{*}{ Silty loamy } & Poultry manure & 145 & 140 & 143 & 30 & 31 & 30.5 & 43 & 44 & 34.5 \\
\hline & Sheep manure & 139 & 150 & 144 & 36 & 37 & 36.5 & 38 & 39 & 38.5 \\
\hline & Cattle manure & 370 & 375 & 373 & 29 & 30 & 29.5 & 52 & 54 & 53.0 \\
\hline & Sewage sludge & 181 & 183 & 182 & 43 & 45 & 44.0 & 47 & 49 & 48.0 \\
\hline & Town waste & 124 & 131 & 128 & 31 & 32 & 31.5 & 32 & 34 & 33.0 \\
\hline & Control(NPK) & 120 & 122 & 121 & 28 & 29 & 28.5 & 43 & 44 & 33.5 \\
\hline & Mean & 180 & 184 & 182 & 33 & 34 & 33.4 & 42.5 & 44 & 43.3 \\
\hline \multirow{7}{*}{$\begin{array}{c}\text { Loamy } \\
\text { sand }\end{array}$} & Poultry manure & 123 & 128 & 126 & 27 & 28 & 27.5 & 29 & 31 & 30.0 \\
\hline & Sheep manure & 117 & 138 & 128 & 23 & 34 & 33.5 & 24 & 26 & 25.0 \\
\hline & Cattle manure & 340 & 350 & 345 & 26 & 28 & 27.0 & 38 & 39 & 38.5 \\
\hline & Sewage sludge & 167 & 168 & 168 & 40 & 41 & 40.5 & 33 & 36 & 34.5 \\
\hline & Town waste & 102 & 109 & 106 & 29 & 30 & 29.5 & 23 & 25 & 34.0 \\
\hline & Control(NPK) & 101 & 104 & 103 & 25 & 26 & 25.5 & 30 & 32 & 31.0 \\
\hline & Mean & 158 & 166 & 162 & 30 & 31 & 30.6 & 29.5 & 30 & 29.8 \\
\hline \multirow[t]{7}{*}{ Sandy } & \begin{tabular}{|l|} 
Poultry manure \\
\end{tabular} & 111 & 117 & 114 & 21 & 22 & 21.50 & 23 & 25 & 24.0 \\
\hline & Sheep manure & 106 & 127 & 117 & 27 & 28 & 27.5 & 18 & 21 & $\mathbf{1 9 . 5}$ \\
\hline & Cattle manure & 324 & 327 & 326 & 20 & 22 & 21.0 & 32 & 34 & 33.0 \\
\hline & Sewage sludge & 143 & 146 & 145 & 34 & 37 & 35.5 & 27 & 29 & 28.0 \\
\hline & Town waste & 93 & 96 & 95 & 23 & 24 & 34.5 & 16 & 19 & $\mathbf{1 7 . 5}$ \\
\hline & Control(NPK) & 91 & 92 & 92 & 20 & 21 & 20.5 & 24 & 26 & 25.0 \\
\hline & Mean & 145 & 151 & 148 & 24 & 28 & 24.9 & 23.3 & 25.7 & 24.5 \\
\hline \multirow{2}{*}{$\begin{array}{c}\text { L.S.D. at } \\
\mathbf{5 \%}\end{array}$} & Soil type (A) & 17.1 & 18.3 & & 10.7 & 11.3 & & 3.6 & 3.7 & \\
\hline & Fertilizer source (B) & 21.4 & 22.3 & & 11.8 & 11.9 & & 3.9 & 3.8 & \\
\hline Interaction & $(\mathbf{A}) \mathbf{X}(\mathbf{B})$ & 26.2 & 26.4 & & 13.4 & 14.9 & & 4.9 & 4.7 & \\
\hline
\end{tabular}

Table (7): Fruit set (\%), June drop (\%) and fruiting (\%)of Valencia orange trees as affected by different sources of organic fertilizer at different soil types.

\begin{tabular}{|c|c|c|c|c|c|c|c|c|c|c|}
\hline \multicolumn{2}{|c|}{ Fruiting parameters } & \multicolumn{3}{|c|}{ Fruit set (\%) } & \multicolumn{3}{|c|}{ June drop (\%) } & \multicolumn{3}{|c|}{ Fruiting (\%) } \\
\hline Soil type (A) & Fertilizer source (B) & 2008 & 2009 & mean & 2008 & 2009 & mean & 2008 & 2009 & mean \\
\hline \multirow[t]{7}{*}{ Silty loamy } & Poultry manure & 28.9 & 29.1 & 29.00 & 66.5 & 65.1 & 65.80 & 1.49 & 1.51 & 1.50 \\
\hline & Sheep manure & 27.8 & 27.9 & 27.85 & 69.1 & 68.8 & 69.00 & 1.34 & 1.36 & 1.35 \\
\hline & Cattle manure & 28.0 & 28.2 & 28.10 & 68.8 & 68.5 & 68.65 & 1.38 & 1.39 & 1.38 \\
\hline & Sewage sludge & 24.7 & 24.9 & 24.80 & 72.1 & 71.5 & 71.80 & 1.28 & 1.29 & 1.29 \\
\hline & Town waste & 24.3 & 24.0 & 24.15 & 77.5 & 76.2 & 76.85 & 1.24 & 1.26 & 1.25 \\
\hline & Control(NPK) & 25.8 & 25.9 & 25.85 & 70.3 & 69.9 & 70.10 & 1.32 & 1.34 & 1.33 \\
\hline & Mean & 26.6 & 26.7 & 26.65 & 70.72 & 70.00 & 70.36 & 1.34 & 1.36 & 1.35 \\
\hline \multirow[t]{7}{*}{ Loamy sand } & Poultry manure & 26.8 & 27.0 & 26.90 & 69.6 & 68.1 & 68.85 & 1.45 & 1.48 & 1.47 \\
\hline & Sheep manure & 25.7 & 25.9 & 25.80 & 72.2 & 71.1 & 71.65 & 1.29 & 1.31 & 1.30 \\
\hline & Cattle manure & 25.9 & 26.1 & 26.00 & 71.7 & 70.5 & 71.10 & 1.33 & 1.35 & 1.34 \\
\hline & Sewage sludge & 22.6 & 22.8 & 22.70 & 75.1 & 74.2 & 74.65 & 1.23 & 1.26 & 1.24 \\
\hline & Town waste & 22.2 & 22.4 & 22.30 & 79.1 & 77.3 & 78.20 & 1.19 & 1.21 & 1.20 \\
\hline & Control(NPK) & 23.7 & 23.9 & 23.80 & 73.3 & 71.4 & 72.35 & 1.27 & 1.29 & 1.28 \\
\hline & Mean & 24.9 & 24.7 & 24.80 & 73.5 & 72.1 & 72.80 & 1.29 & 1.32 & 1.31 \\
\hline \multirow[t]{7}{*}{ Sandy } & Poultry manure & 22.5 & 22.7 & 22.60 & 71.5 & 70.3 & 70.90 & 1.40 & 1.42 & 1.41 \\
\hline & Sheep manure & 21.4 & 21.6 & 21.50 & 74.1 & 73.2 & 73.65 & 1.25 & 1.28 & 1.27 \\
\hline & Cattle manure & 21.6 & 21.8 & 21.70 & 73.4 & 72.5 & 72.95 & 1.29 & 1.32 & 1.31 \\
\hline & Sewage sludge & 18.1 & 18.7 & $\overline{18.40}$ & 77.1 & 75.2 & 76.10 & 1.19 & 1.21 & 1.20 \\
\hline & Town waste & 18.3 & 18.9 & 18.60 & 82.1 & 79.4 & 80.75 & 1.15 & 1.19 & 1.17 \\
\hline & Control(NPK) & 19.4 & 20.2 & 19.80 & 75.4 & 73.2 & 74.30 & 1.23 & 1.26 & 1.24 \\
\hline & Mean & 20.22 & 20.65 & 20.44 & 75.6 & 73.9 & 74.80 & 1.25 & 1.28 & 1.27 \\
\hline \multirow[t]{2}{*}{ L.S.D. at 5\% } & Soil type (A) & 0.29 & 0.31 & & 0.21 & 0.23 & & 0.07 & 0.06 & \\
\hline & Fertilizer source (B) & 0.43 & 0.49 & & 0.34 & 0.41 & & 0.09 & 0.11 & \\
\hline Interaction & $(\mathbf{A}) \mathbf{X}(\mathrm{B})$ & 0.84 & 0.76 & & 0.87 & 0.93 & & $\mathbf{0 . 4 3}$ & 0.44 & \\
\hline
\end{tabular}

Fayoum J. Agric. Res. \& Dev., Vol.25, No.2, July, 2011 
RESPONSE OF VALENCIA ORANGE TREES TO..........

Yield:

Results concerning yield (fruit number per tree, kgs per tree and Tons per feddan) are shown in Table (8). The obtained data revealed that fertilizing Valencia orange trees with organic and mineral fertilizers were most effective in increasing yield. The main observation is that treating trees with poultry manure fertilizer had the bigger yield than those treated with other fertilizer sources. Moreover, second season gave higher yield than the first season in all organic or mineral fertilizers. Nevertheless, there were significant differences between fertilizer sources as well as between soil types in yield. Also, the interaction between fertilizer sources and soil types was significant.

These results were agreed with Motskobili, (1984). and Huang, et. al., (1995). On Satsuma mandarin trees mentioned that using organic manure gave satisfactory increase in yield

Table (8): Yield of Valencia orange trees as affected by different sources of organic fertilizer at different soil types.

\begin{tabular}{|c|c|c|c|c|c|c|c|c|c|c|}
\hline \multicolumn{2}{|c|}{$\begin{array}{l}\text { Yield parameters } \\
\end{array}$} & \multicolumn{3}{|c|}{ Fruit No. /tree } & \multicolumn{3}{|c|}{ Yield/tree(kg) } & \multicolumn{3}{|c|}{ Yield/feddan (Ton) } \\
\hline Soil type (A) & Fertilizer source (B) & 2008 & 2009 & mean & 2008 & 2009 & mean & 2008 & 2009 & mean \\
\hline \multirow[t]{7}{*}{ Silty loamy } & Poultry manure & 326 & 329 & 328 & 81.21 & 82.81 & 82.01 & 13.12 & 13.64 & 13.38 \\
\hline & Sheep manure & 315 & 317 & 316 & 70.91 & 70.37 & 70.64 & 11.82 & 11.91 & $\mathbf{1 1 . 8 7}$ \\
\hline & Cattle manure & 320 & 322 & 321 & 74.21 & 75.79 & 75.00 & 12.46 & 12.73 & 12.60 \\
\hline & Sewage sludge & 300 & 302 & 301 & 60.69 & 62.94 & 61.81 & 10.19 & 10.54 & 10.37 \\
\hline & Town waste & 290 & 297 & 293 & 56.84 & 59.69 & 58.19 & 9.55 & 10.03 & 9.78 \\
\hline & Control(NPK) & 305 & 307 & 306 & 66.79 & 67.85 & 61.41 & 11.22 & 11.39 & 11.30 \\
\hline & Mean & 309 & 312 & 311 & 68.44 & 69.91 & 69.18 & 11.49 & 11.61 & 11.55 \\
\hline \multirow[t]{7}{*}{ Loamy sand } & Poultry manure & 320 & 323 & 321 & 76.22 & 77.55 & 76.89 & 12.80 & 13.03 & 12.91 \\
\hline & Sheep manure & 309 & 311 & 310 & 66.15 & 67.52 & 66.84 & 11.11 & 11.34 & 11.22 \\
\hline & Cattle manure & 314 & 317 & 315 & 69.33 & 70.98 & 70.15 & 11.65 & 11.92 & 11.78 \\
\hline & Sewage sludge & 294 & 298 & 296 & 56.30 & 57.99 & 57.14 & 9.45 & 9.74 & 9.60 \\
\hline & Town waste & 284 & 288 & 286 & 52.27 & 54.48 & 53.38 & 8.78 & 9.15 & 8.96 \\
\hline & Control(NPK) & 299 & 301 & 300 & 62.19 & 63.51 & 62.85 & 10.45 & 10.66 & 10.55 \\
\hline & Mean & 303 & 306 & 305 & 63.74 & 65.34 & 64.54 & 10.71 & 10.97 & 10.84 \\
\hline \multirow[t]{7}{*}{ Sandy } & Poultry manure & 318 & 320 & 319 & 74.13 & 76.54 & 75.34 & $\mathbf{1 2 . 4 5}$ & 12.86 & 12.65 \\
\hline & Sheep manure & 307 & 309 & 308 & 64.19 & 65.53 & 64.86 & 10.77 & 11.01 & 10.89 \\
\hline & Cattle manure & 313 & 315 & 314 & 67.57 & 69.07 & 68.32 & 11.35 & 11.60 & 11.47 \\
\hline & Sewage sludge & 292 & 295 & 293 & 54.46 & 56.02 & 55.24 & 9.15 & 9.41 & 9.28 \\
\hline & Town waste & 282 & 286 & 284 & 50.76 & 52.94 & 51.85 & 8.53 & 8.89 & 8.71 \\
\hline & Control(NPK) & 297 & 301 & 299 & 60.29 & 62.94 & 61.62 & 10.13 & 10.57 & $\mathbf{1 0 . 3 5}$ \\
\hline & Mean & 302 & 304 & 303 & 61.90 & 63.84 & 62.87 & 10.39 & 10.72 & 10.56 \\
\hline \multirow[t]{2}{*}{ L.S.D. at 5\% } & Soil type (A) & 2.9 & 2.8 & & 2.7 & 2.6 & & 0.15 & 0.19 & \\
\hline & Fertilizer source (B) & 2.7 & 2.6 & & 2.5 & 2.4 & & 0.21 & 0.24 & \\
\hline Interaction & $(\mathbf{A}) \mathbf{X}(\mathbf{B})$ & 2.99 & 3.11 & & 2.66 & 2.69 & & 0.43 & 0.49 & \\
\hline
\end{tabular}

Fruit Quality:

\section{1-Fruit physical properties:}

Data presented in Table (9) cleared that the studied parameters including fruit weight $(\mathrm{gm})$, fruit size $\left(\mathrm{cm}^{3}\right)$ and juice $\%$ had significant differences in all fertilizers sources. Trees received organic fertilizers such as Poultry manure, cattle manure, sheep manure, chemical fertilizer, sewage sludge, town waste, respectively improved fruit physical properties. Trees treated with town waste had the lowest fruit physical properties. The interaction between fertilizer sources and soil types was significant.

Fayoum J. Agric. Res. \& Dev., Vol.25, No.2, July, 2011 
Table (9): Some fruit physical properties of Valencia orange trees as affected by different sources of organic fertilizer at different soil types.

\begin{tabular}{|c|c|c|c|c|c|c|c|c|c|c|}
\hline \multicolumn{2}{|c|}{ Some fruit physical properties } & \multicolumn{3}{|c|}{ Fruit weight (gm) } & \multicolumn{3}{|c|}{ Fruit $\operatorname{size}\left(\mathrm{cm}^{3}\right)$} & \multicolumn{3}{|c|}{ Juice (\%) } \\
\hline Soil type (A) & Fertilizer source (B) & 2008 & 2009 & mean & 2008 & 2009 & mean & 2008 & 2009 & mean \\
\hline \multirow[t]{7}{*}{ Silty loamy } & Poultry manure & 249.1 & 251.7 & 250.4 & 199.0 & 201.0 & 200.0 & $\mathbf{5 7 . 0}$ & 53.0 & 55.0 \\
\hline & Sheep manure & 225.1 & 222.0 & 223.5 & 192.0 & 194.0 & 193.0 & 54.0 & 51.0 & 54.5 \\
\hline & Cattle manure & 231.9 & 235.4 & 233.7 & 196.0 & 197.0 & 196.5 & 55.0 & 52.0 & 53.5 \\
\hline & Sewage sludge & 202.5 & 208.4 & 205.5 & 188.0 & 189.0 & 188.5 & 52.0 & 49.0 & 50.4 \\
\hline & Town waste & 196.0 & 201.0 & 198.5 & 184.0 & 185.0 & 184.5 & 48.0 & 44.0 & 46.0 \\
\hline & Control(NPK) & 219.0 & 221.0 & 220.0 & 190.0 & 191.0 & 190.5 & 53.0 & 49.0 & 51.0 \\
\hline & Mean & 220.6 & 223.3 & 221.9 & 192.9 & 192.8 & 192.9 & 53.2 & 49.7 & 51.6 \\
\hline \multirow[t]{7}{*}{ Loamy sand } & \begin{tabular}{|l|} 
Poultry manure \\
\end{tabular} & 238.2 & 240.1 & 239.1 & 196.0 & 198.0 & 197.0 & 55.0 & 51.0 & 53.0 \\
\hline & Sheep manure & 214.1 & 217.1 & 215.6 & 190.0 & 191.0 & 190.5 & 52.0 & 48.0 & 50.0 \\
\hline & Cattle manure & 220.8 & 223.9 & 222.4 & 193.0 & 194.0 & 193.5 & 53.0 & 49.0 & 51.0 \\
\hline & Sewage sludge & 191.5 & 194.6 & 193.1 & 184.0 & 185.0 & 184.5 & 50.0 & 46.0 & 48.0 \\
\hline & Town waste & 185.1 & 189.2 & 187.1 & 181.0 & 182.0 & 181.8 & 46.0 & 41.0 & 43.5 \\
\hline & Control(NPK) & 208.0 & 211.0 & 209.5 & 188.0 & 189.0 & 188.5 & 51.0 & 48.0 & 49.5 \\
\hline & Mean & 209.6 & 212.7 & 211.2 & 188.6 & 189.8 & 189.2 & 51.2 & 47.2 & 49.2 \\
\hline \multirow[t]{7}{*}{ Sandy } & Poultry manure & 233.1 & 239.2 & 236.1 & 193.0 & 194.0 & $\mathbf{1 9 3 . 5}$ & 54.0 & 50.0 & 52.0 \\
\hline & Sheep manure & 209.1 & 212.1 & 210.5 & 188.0 & 189.0 & 188.5 & 51.0 & 47.0 & 48.0 \\
\hline & Cattle manure & 215.9 & 219.3 & 217.6 & 191.0 & 190.0 & 190.5 & 52.0 & 58.0 & 55.0 \\
\hline & Sewage sludge & 186.5 & 189.9 & 188.2 & 182.0 & 180.0 & 181.0 & 49.0 & 45.0 & 47.0 \\
\hline & Town waste & 180.0 & 185.1 & 182.5 & 179.0 & 180.0 & 178.5 & 45.0 & 41.0 & 43.0 \\
\hline & Control(NPK) & 203.0 & 209.1 & 206.1 & 185.0 & 186.0 & 185.5 & 50.0 & 46.0 & 48.0 \\
\hline & Mean & 204.6 & 209.1 & 206.9 & 186.3 & 186.5 & 186.4 & 50.2 & 47.8 & 49.0 \\
\hline \multirow{2}{*}{ 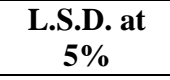 } & \begin{tabular}{|l|} 
Soil type (A) \\
\end{tabular} & 1.7 & 1.5 & & 1.8 & 1.7 & & 0.28 & 0.31 & \\
\hline & Fertilizer source (B) & 1.9 & 1.8 & & 1.9 & 1.8 & & $\mathbf{0 . 3 5}$ & 0.48 & \\
\hline Interaction & \begin{tabular}{|c|}
$(\mathbf{A}) \mathbf{X}(\mathbf{B})$ \\
\end{tabular} & 1.44 & 1.49 & & 1.22 & 1.37 & & 0.74 & 0.79 & \\
\hline
\end{tabular}

2- Fruit chemical properties:

Data illustrated in Table (10) showed that the studied parameters i.e., T.S.S.\%, T.S.S./ acid ratio and ascorbic acid $(\mathrm{mg} / 100 \mathrm{ml}$ juice) were increased by fertilizing with organic or mineral sources but organic manure sources were effective in this respect. The improvement in fruit quality in response to application of organic manure and mineral fertilizers could be arranged in descending order as follows: poultry manure, cattle manure, sheep manure, chemical fertilizer, sewage sludge and town waste was noticed. These results were recorded in all studied soil types and the interaction between fertilizer sources and soil types was significant. These results agree with those obtained by Motskobili, (1984). on Satsuma mandarin, Chokha, et. al., (1993). on Sweet orange trees, Huang, et. al., (1995). on Satsuma mandarin trees, El- Kobbiaobbia(1999). on Navel orange trees, Grassi, et. al., (1999). on Rangpour Lime, and Helail, et. al., (2003). on Washington navel orange trees mentioned that using organic manure was improving fruit quality and fruits had more T.S.S.\% and ascorbic acid. 
RESPONSE OF VALENCIA ORANGE TREES TO.........

Table (10): Some fruit chemical properties of Valencia orange trees as affected by different sources of organic fertilizer at different soil types.

\begin{tabular}{|c|c|c|c|c|c|c|c|c|c|c|c|c|c|}
\hline \multicolumn{2}{|c|}{ Some fruit chemical properties } & \multicolumn{3}{|c|}{$\begin{array}{l}\text { T.S.S. } \\
(\%)\end{array}$} & \multicolumn{3}{|c|}{ Acidity (\%) } & \multicolumn{3}{|c|}{ T.S.S./acid ratio } & \multicolumn{3}{|c|}{$\begin{array}{c}\text { Ascorbic acid } \\
\text { (mg/100ml juice) }\end{array}$} \\
\hline Soil type $(A)$ & $\begin{array}{l}\text { Fertilizer source } \\
\text { (B) }\end{array}$ & 2008 & 2009 & mean & 2008 & 2009 & mean & 2008 & 2009 & mean & 2008 & 2009 & mean \\
\hline \multirow[t]{7}{*}{ Silty loamy } & Poultry manure & 12.7 & 12.8 & 12.75 & 1.1 & 1.2 & 1.15 & 11.54 & 10.67 & 11.11 & 46.0 & 48.0 & 47.00 \\
\hline & Sheep manure & 12.1 & 12.3 & 12.20 & 1.1 & 1.1 & 1.10 & 11.00 & 11.18 & 11.09 & 40.1 & 40.8 & 40.45 \\
\hline & Cattle manure & 12.4 & 12.6 & 12.50 & 1.1 & 1.1 & 1.10 & 11.27 & 11.45 & 11.36 & 43.0 & 44.0 & 43.50 \\
\hline & Sewage sludge & 11.8 & 11.9 & 11.85 & 1.1 & 1.1 & 1.10 & 10.73 & 10.82 & 10.78 & 36.0 & 37.0 & 36.50 \\
\hline & Town waste & 11.6 & 11.8 & 11.70 & 1.2 & 1.2 & 1.20 & 9.66 & 9.83 & 9.75 & 34.4 & 35.1 & 34.75 \\
\hline & Control(NPK) & 11.9 & \begin{tabular}{|l|}
12.1 \\
\end{tabular} & 12.00 & 1.1 & 1.1 & 1.10 & 10.82 & 11.00 & 10.91 & 39.2 & 41.0 & 40.10 \\
\hline & Mean & 12.08 & 12.25 & 12.17 & 1.1 & 1.1 & 1.10 & 10.89 & 10.99 & 10.94 & 39.8 & 40.9 & 40.38 \\
\hline \multirow[t]{7}{*}{ Loamy sand } & Poultry manure & 12.5 & \begin{tabular}{|l|l|}
12.7 \\
\end{tabular} & 12.60 & 1.1 & 1.1 & 1.10 & 11.36 & 11.54 & 11.45 & 45.0 & 45.2 & 45.10 \\
\hline & Sheep manure & 11.9 & 12.2 & 11.05 & 1.1 & 1.1 & 1.10 & 10.81 & 11.09 & 10.95 & 39.3 & 40.1 & 39.70 \\
\hline & Cattle manure & 12.2 & 12.5 & 12.35 & 1.1 & 1.1 & 1.10 & 11.09 & 11.36 & 11.23 & 42.1 & 42.7 & 42.40 \\
\hline & Sewage sludge & 11.6 & 11.7 & 11.65 & 1.0 & 1.0 & 1.00 & 11.60 & 11.70 & 11.65 & 35.2 & 35.9 & 35.55 \\
\hline & Town waste & 11.4 & 11.6 & 11.50 & 1.2 & 1.2 & 1.20 & 9.50 & 9.67 & 9.59 & 33.1 & 33.8 & 33.45 \\
\hline & Control(NPK) & 11.7 & 11.9 & 11.80 & 1.1 & 1.1 & 1.10 & 10.64 & 10.64 & 10.63 & 37.9 & 38.4 & 38.15 \\
\hline & Mean & 11.9 & 12.1 & 12.00 & 1.1 & 1.1 & 1.10 & $\mathbf{1 0 . 8 3}$ & 10.99 & 10.91 & 38.8 & 39.4 & 39.1 \\
\hline \multirow[t]{7}{*}{ Sandy } & Poultry manure & 12.1 & 12.4 & 12.25 & 1.1 & 1.1 & 1.10 & 11.00 & 11.27 & 11.14 & 43.8 & 44.2 & 44.0 \\
\hline & Sheep manure & 11.5 & 11.8 & 11.65 & 1.1 & 1.1 & 1.10 & 10.45 & 10.73 & 10.59 & 38.1 & 38.8 & 38.45 \\
\hline & Cattle manure & 11.8 & 12.1 & 11.45 & 1.1 & 1.1 & 1.10 & 10.73 & 11.00 & 10.87 & 41.2 & 41.7 & 41.45 \\
\hline & Sewage sludge & 11.2 & 11.6 & 11.40 & 1.0 & 1.1 & 1.05 & 11.20 & 10.54 & 10.86 & 34.0 & 34.8 & 34.40 \\
\hline & Town waste & 11.0 & 11.4 & 11.20 & 1.1 & 1.2 & 1.15 & 10.00 & 9.50 & 9.75 & 32.1 & 32.5 & 32.30 \\
\hline & Control(NPK) & 11.3 & 11.5 & 11.40 & 1.1 & 1.1 & 1.10 & 10.27 & 10.45 & 10.36 & 36.3 & 36.9 & 36.60 \\
\hline & Mean & 11.5 & 11.8 & 11.70 & 1.1 & 1.1 & 1.10 & 10.61 & 10.58 & 10.60 & 37.6 & 38.2 & 37.90 \\
\hline \multirow[t]{2}{*}{ L.S.D. at 5\% } & \begin{tabular}{|l|} 
Soil type (A) \\
\end{tabular} & 0.7 & 0.6 & & 0.04 & 0.05 & & 0.5 & 0.04 & & 0.29 & 0.32 & \\
\hline & Fertilizer source $(\mathrm{B})$ & 0.8 & 0.9 & & 0.06 & 0.08 & & 0.13 & 0.17 & & 0.34 & 0.42 & \\
\hline Interaction & $(\mathbf{A}) \mathbf{X}(\mathbf{B})$ & 1.02 & 1.04 & & 0.11 & 0.14 & & 0.22 & 0.24 & & 0.73 & 0.79 & \\
\hline
\end{tabular}

\section{Soil content of different nitrogen fractions:}

Table (11) cleared that total nitrogen $(\mathrm{N})$ and $\left(\mathrm{NH}_{4}\right)$ remained in the soil was positively affected by the source of $\mathrm{N}$ fertilizer (Organic or mineral). The highest Total $\mathrm{N}$ values were obtained by organic manure i.e. poultry manure, cattle manure, sheep manure, mineral fertilizer, sewage sludge and town waste in descending order. Soil $\mathrm{No}_{3}$ content as a results of fertilization, chemical fertilizer was positively affected than organic manure while, NH4 values were obtained by using poultry manure, sheep manure, cattle manure, sewage sludge, mineral and town waste in descending order. Soil fertilized with poultry manure had the largest $\mathrm{No}_{3}$ in organic fertilizers followed by sheep \& sewage manure then town waste whereas, soil treated with cattle manure recorded the lowest $\mathrm{No}_{3}$ These results were true in all studied soils. The values of these results were greater in silty loam, sandy loam and sandy soil in a descending order.

The superiority of organic manure fertilizer may be due to slow leaching from soil which lead to higher efficiency. Besides, the favorable effects of poultry manure may be associated with its action reducing soil PH and subsequently enhancing the uptake of nutrients. The beneficial effect of organic manure on amending the trees with their requirements from $\mathrm{N}$ at longer period in addition to the lower loss of $\mathrm{N}$ applied of organic fertilizers could explain the present results. So, exhibits total $\mathrm{N}$ remained in the soil for a long time.

Fayoum J. Agric. Res. \& Dev., Vol.25, No.2, July, 2011 
Table 11

Fayoum J. Agric. Res. \& Dev., Vol.25, No.2, July, 2011 
Some soil physical and chemical properties:

Data of Table (12) indicated that organic fertilizers were more effective than chemical fertilizer in improving the soil characteristics. Soil organic matter content (OM) significantly increased, while, the soil PH were decreased as a results of organic fertilizers addition. The decrease in soil $\mathrm{pH}$ is due to organic acids produced during organic manure decomposition and its effects on solubility of some soil minerals or due to releasing the nutrients through organic manure decay by microorganism activity. Data presented in the same Table revealed that applying of organic manure significantly increased the level of available nutrients in soil. These increase due to the increase of acidity produced by adding of the organic manures decomposition and its effects on solubility of some soil minerals. The high level of soil $\mathrm{N}$, and $\mathrm{P}$ were found with the addition of poultry manure while, using cattle manure led to increase soil (K, Fe and $\mathrm{Mn}$ ) contents. Adding sewage sludge compost to the soil led to increase the soil ( $\mathrm{ZN}$ and $\mathrm{Cu})$ contents than the other fertilizers.

Data cleared also, that silty loam soil had the highest contents of nitrogen and phosphorus as a results of treatments, while, loamy sand recorded the highest values of $\mathrm{K}, \mathrm{Fe}, \mathrm{Zn}, \mathrm{Mn}$ and $\mathrm{Cu}$. Sandy soil had the lowest contents of these elements.

Table (12): Soil OM ,PH and some available nutrients at different soil types as affected by different sources of organic fertilizer at the end of experiment.

\begin{tabular}{|c|c|c|c|c|c|c|c|c|c|}
\hline \multirow{2}{*}{\multicolumn{2}{|c|}{\begin{tabular}{|l|l} 
Soil type (A) & Fertilizer source (B)
\end{tabular}}} & \multirow{2}{*}{$\begin{array}{l}\text { OM } \\
(\%)\end{array}$} & \multirow{2}{*}{$\mathbf{P H}$} & \multicolumn{6}{|c|}{ Available soil nutrients (mg/kg soil) } \\
\hline & & & & $\mathbf{P}$ & $\mathbf{K}$ & $\mathbf{F e}$ & $\mathbf{Z n}$ & Mn & $\mathrm{Cu}$ \\
\hline \multicolumn{2}{|c|}{ available nutrients before addition } & $\mathbf{1 . 8 6}$ & 8.45 & 21.4 & 22.5 & 2.4 & $\mathbf{0 . 8}$ & 1.9 & $\mathbf{0 . 3 3}$ \\
\hline \multirow{7}{*}{ Silty loamy } & Poultry manure & 2.31 & 8.20 & 25.4 & 35.9 & $\mathbf{3 . 3}$ & 1.3 & 2.8 & 0.90 \\
\hline & Sheep manure & 2.12 & 8.28 & 22.3 & 35.6 & 3.9 & 1.5 & 2.7 & 0.90 \\
\hline & Cattle manure & 1.94 & 8.32 & 20.1 & 37.2 & 4.3 & 1.1 & 3.4 & 0.80 \\
\hline & Sewage sludge & 1.94 & 8.32 & 20.5 & 35.4 & 3.7 & 1.6 & 3.0 & 1.20 \\
\hline & Town waste & 1.96 & 8.40 & 19.3 & 35.9 & 3.5 & 1.2 & 2.6 & 1.00 \\
\hline & Control (NPK) & 1.93 & 8.00 & 18.2 & 24.7 & 3.2 & 1.1 & 2.5 & 0.90 \\
\hline & Mean & 2.03 & 8.20 & 20.9 & 34.1 & 3.7 & 1.3 & 2.83 & 0.95 \\
\hline \multicolumn{2}{|c|}{ available nutrients before addition } & 0.80 & 8.01 & 18.3 & 23.2 & 3.8 & 1.6 & 2.9 & 1.1 \\
\hline \multirow[t]{7}{*}{ Loamy sand } & Poultry manure & 2.08 & 7.83 & 22.3 & 45.9 & 3.9 & 1.7 & 3.2 & $\mathbf{1 . 0}$ \\
\hline & Sheep manure & 1.89 & 7.89 & 19.2 & 43.8 & 4.5 & 1.8 & 3.1 & 1.0 \\
\hline & Cattle manure & 1.71 & 7.93 & 17.0 & 44.5 & 4.9 & 1.4 & 3.8 & 0.9 \\
\hline & Sewage sludge & 1.71 & 7.91 & 17.4 & 42.7 & 4.3 & 1.9 & 3.4 & 1.3 \\
\hline & Town waste & 1.63 & 7.96 & 16.2 & 43.2 & 4.1 & 1.5 & 3.0 & 1.1 \\
\hline & Control(NPK) & 1.50 & 7.77 & 15.1 & 32.0 & 3.8 & 1.4 & 2.9 & 1.0 \\
\hline & Mean & 1.75 & 7.88 & 17.9 & 42.0 & 4.3 & 1.6 & 3.2 & 1.06 \\
\hline \multicolumn{2}{|c|}{ available nutrients before addition } & 0.04 & 7.85 & 5.6 & 16.19 & 1.14 & 0.41 & 0.16 & 0.8 \\
\hline \multirow[t]{7}{*}{ Sandy } & Poultry manure & 1.71 & 7.72 & 18.1 & 32.5 & 3.1 & 1.1 & 2.4 & 0.8 \\
\hline & Sheep manure & 1.52 & 7.74 & 15.0 & 32.2 & 3.7 & 1.3 & 2.5 & 0.8 \\
\hline & Cattle manure & 1.34 & 7.78 & 12.8 & 33.8 & 4.1 & 0.9 & 3.2 & 0.7 \\
\hline & Sewage sludge & 1.33 & 7.77 & 13.2 & 32.0 & 3.5 & 1.4 & 2.8 & 1.1 \\
\hline & Town & 1.26 & 7.81 & 12.0 & 32.4 & 3.2 & 1.0 & 2.4 & 0.9 \\
\hline & Contro & 1.13 & 7.68 & 11.9 & 21.3 & 3.0 & 0.9 & 2.3 & 0.8 \\
\hline & & 1.38 & 7.75 & 13.8 & 30.7 & 3.4 & 1.1 & 2.6 & 0.9 \\
\hline \multirow[t]{2}{*}{ L.S.D. at \% } & Soil typ & 0.77 & 0.7 & 0.04 & 0.06 & 0.09 & 0.05 & 0.26 & 0.38 \\
\hline & Fertilizer source (B) & 0.83 & 0.8 & 0.07 & 0.07 & 0.14 & 0.16 & 0.31 & 0.46 \\
\hline Interaction & $(\mathrm{A}) \mathbf{X}(\mathrm{B})$ & 1.07 & 1.09 & 0.18 & 0.16 & \begin{tabular}{|l|}
0.27 \\
\end{tabular} & 0.28 & 0.74 & 0.81 \\
\hline
\end{tabular}

These results are in harmony with those obtained by Abou seeda (1997), Fliessbach et al; (2000) and Abdel Nasser and Harhash (2000). They concluded that, applying organic manure to soil for reducing soil $\mathrm{pH}$ led to increase $\mathrm{N}, \mathrm{P}, \mathrm{K}, \mathrm{Fe}$, $\mathrm{Zn}$ and Mn soil contents. Moreover, organic manure improves the water holding capacity, cation exchange capacity and stabilizes the properties of sandy soils.

Fayoum J. Agric. Res. \& Dev., Vol.25, No.2, July, 2011 
Organic matter also improves the nutritional status of all the soil types and sandy soil in particular. Finally, they suggested that organic manure application increased the transfer of elements between solid phase and soil solution by the higher microbial activity.

\section{CONCLUSION:}

Egyptian soils are poor in organic matter. Using organic manure as a source of $\mathrm{N}$ requirements for trees production reduced fertilization costs and decreasing the losses in total $\mathrm{N}$ that can cause by leaching of nitrate or reduction of nitrate resulting in the formation of gaseous nitrogen that loss by volatilization when using chemical fertilizers. Thus, using organic manure considered as an alternative source of trees nutrients as well as a soil amendment to improve the soil physical properties that enhance the tree production.

Generally, it could be concluded that there is a strong positive correlation between using organic fertilizer and the improving of vegetative growth leaf water, chlorophyll and mineral contents. Significant decrease of June drop which led to significant increase in yield/tree are also obtained..

Conclusively, adding organic fertilizer plays an active and important role for improving the soil organic matter and nutrients.

Finally, poultry manure addition attained great significant effect on tree growth, higher yield and better fruit quality (T.S.S ,T.S.S/acid ratio and ascorbic acid content). In conclusion, the presented investigation demonstrated the validity of producing Valencia orange trees using organic manure as the sole nutrients supplier, The composted sources, generally showed better growth and increase in leaf pigments and mineral contents as well as greater fruit set and fruiting which led to greater yield, fruit weight, size and improved most fruit quality components as compared with NPK fertilizers. The addition of organic manure improved the organic matter and decreased $\mathrm{pH}$ which led to releasing the nutrient and increase $\mathrm{N}, \mathrm{P}, \mathrm{K}, \mathrm{Fe}, \mathrm{Zn}$ ,Mn and $\mathrm{Cu}$ soil contents.

\section{REFERENCES}

Abdel-Nasser. G. and M.M. Harhash (2000): Effect of organic manures in combination with elemental sulphur on soil physical and chemical characteristics, yield, fruit quality, leaf water contents and nutritional status of flame seedlees grapevine I. Soil physical and chemical characteristics. J. Agric. Sci. Mansoura Univ; 25 (6): 3541-3558.

Abou Sayed- Ahmed, T.A. (1997): Growth and fruiting of Baladi mandarin trees in relation to some soil fertilization treatments in sandy soil. 2-leaf and root responses to the applied treatments. Zagazig J. Agric. Res., 24(6): 1049-1063.

Abou Seeda, M.A. (1987): Chemical and environmental aspects of Sewage Sludge application on Egyptian soils. Ph. Thesis state univ. Gent.,Belgium..

A.O.A.C. (1975): Official methods of analysis. 12 th Ed. Association of Official Analysis Chemists, Washington, D.C.

Balba, A.M. (1976): Soil fertility and fertilization. Dar El-Matboly El-Gadida Alex. Egypt.

Brown, J.D. and O.L., Lilliland (1946): Rapid determination of potassium and sodium in plant material and soil extracts by flame photometery. Proc. Amer. Soc. Hort. Sic; 48:341- 346.

Chapman, H.D. and P.F., Pratt (1961): Methods of analysis for soils, plants and waters. Univ. of California, Div. Agric. Sci; 60.

Fayoum J. Agric. Res. \& Dev., Vol.25, No.2, July, 2011 
RESPONSE OF VALENCIA ORANGE TREES TO.

Chokha, Sing;B.B.Sharma and C.T., Sing (1993): Leaf nutrient composition of sweet orange as affected by combined use of organic and chemical fertilizers. South Indian;41(3):131-134.

El-Aila, H.I.,S. El-Ashry and M. Abo-Seeda, (2001): Studies on a complex slow release fertilizers; II. Ionic compound astablished through interaction between essential micronutrients and urea and their effects on lettuce plant. J. Agric. Sci. Mansoura Univ., 27(6):4311-4324.

El-Kobbiaobbia, A.M. (1999): Response of Wasington navel orange trees to organic fertilizer "biohumus" and cattle manure application. Alex. J. of Agric. Res; 44 (2): 199-207.

Fliessbach, A.; P. Mader, D. Dubois and L. F.Gunst (2000): Results from a 21 year old field trial. Organic farming enhances soil fertility and biodiversity. FIBL- Dossier No 1, 15 pp19.

Grassi, Filho, H.; Pereira, M.A.; Savino, A.A. and V.T. Rodrigues, (1999): Growth of Rangpour Lime seedlings on different substrates. Revista Brasileira de Fruitculture, 21(2):186-190.

Helail, B.M.; Y.N. Gobran, and M.H. Moustafa (2003): Study on the effect of organic manure source, method of organic manure application and biofertilizers on tree growth, leaf mineral contents, fruiting and fruit quality of Wasington navel orange trees. Egypt J. Appl. Sci; 18(4A) 297-320.

Holden, M.L. (1965): Chlorophyll's In Chemistry and biochemistry of plant pigments. Ed. Goodwin, TWmP.P.4. 62-88. Academic press, London.

Huang, F.S.; H.S. Zhang and N.Q. Qian (1995): Study on the effect of organic manures and leaf-spraying P and K on the fruit quality of extra early Satsuma mandarin. China citrus, 24(2):31-32.

Jackson, M.L. (1958): Soil chemical analysis. Prentice- Hall of India, PP. 144-197.: 424-434.

Motskobili, N.A. (1984): Assimilation area of Satsuma in relation to mineral nutrients and its effect on productivity. Subtropicheskie Kultury, No.5.83-90.

Pregl, E. (1945): Quantitative organic micro- analysis 4 Ed; J. Churchill, London. 549-600.

Snedecor, G.W. and W.G., Cochran (1985): Statistical Methods. The Lowa State Univ. Press; Ames, Iowa, USA.

Unemya, Y and A.S.,Sekiya (1985): Effect of heavy application of animal manure on soil chemical properties, leaf nutrients composition and fruit quality in orchards. Bulletin, fruit tree Res. Stat. of Japan, 12:61-78

Vyvyan, M.C. (1946): Experiments with growth substance, sprays for reduction of preharvest drop on fruit. J. Pomol; 22:11-37.

Warr, J.F. and S.B. Hormick, (1990): Recent development in alternative agricultural in the united states. In Proc. of Inthl. Conf. on Kyussei. Nature forming. October 17- 21,1989, Khonkean Univ. Thailand.

Watson, D.J. (1985): The dependence of net assimilation rate of leaf area index. Ann Bot. Lond N.S.:37-54

Weatherley, P.E. (1950): Studies on the water relations of the plant ; I- the field measurements of water in leaves, New Phytologist 49:81-97

Wilde, S.A., Lyer, R.B. and G.K., Voiget (1985): "Soil and plant analysis for free culture" Published by Oxford and IBM Publishing Cooperation, New Delhi, India,pp.93-105.

Fayoum J. Agric. Res. \& Dev., Vol.25, No.2, July, 2011 
Ensherah A.H.Tayeh, et. al.

أستجابة أشجار البرتقال الفالنشيا لبعض مصادر الأسمدة العضوية تحت أنواع مختلفة من التربة

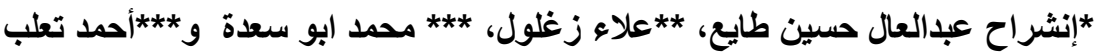

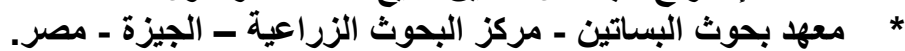

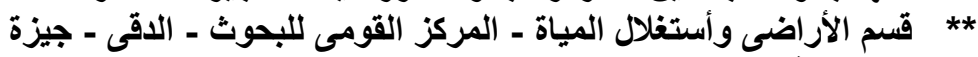

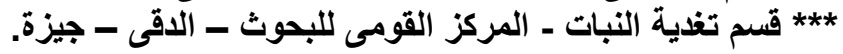

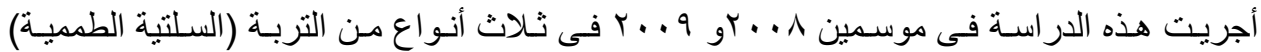

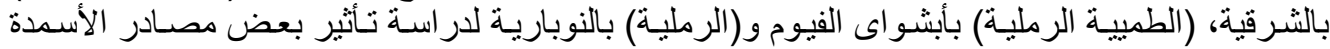

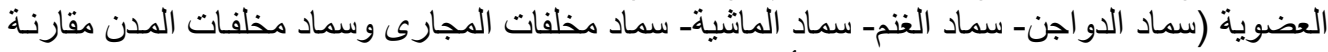

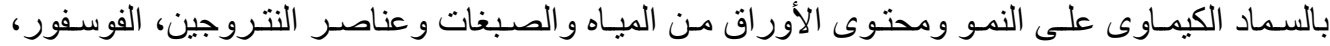

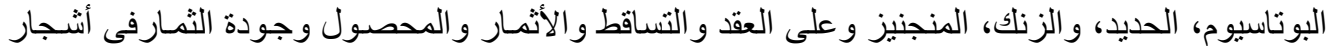

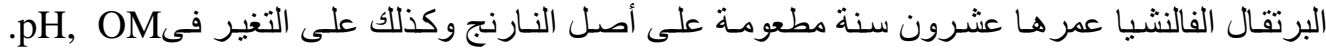

ومحتوى التربة من (N, NH4, No3, P, K, Fe, Mn, Zn and Cu)

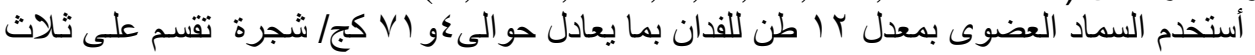

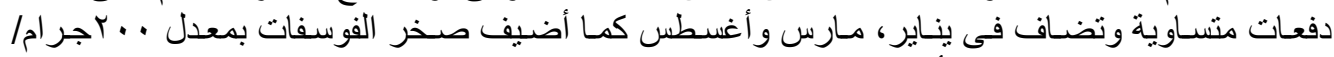

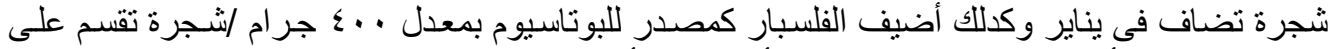

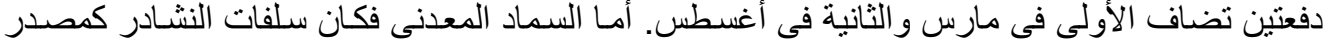

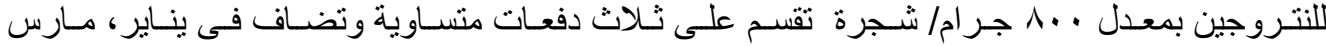

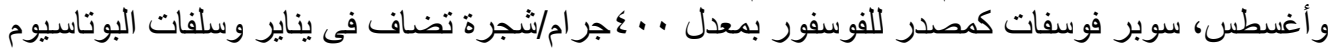

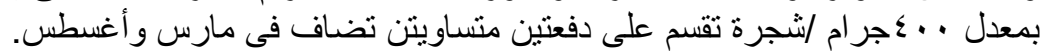

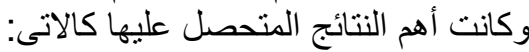

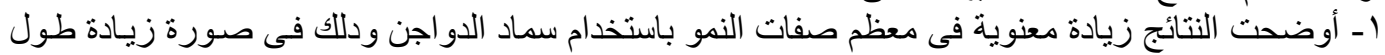

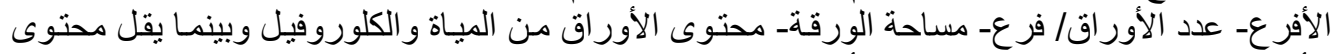

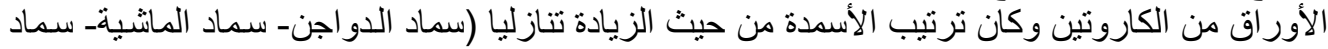

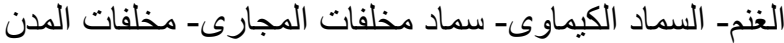

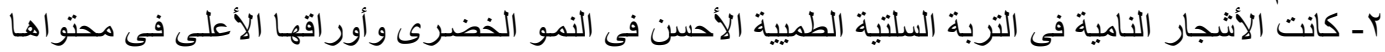

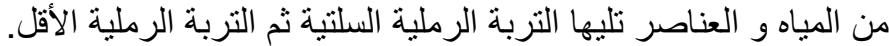

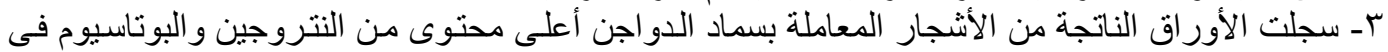

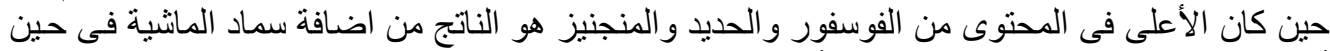

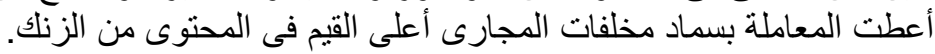

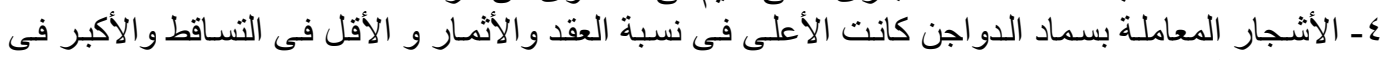

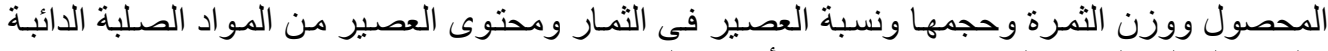

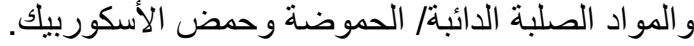

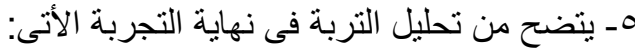

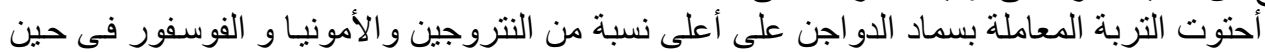

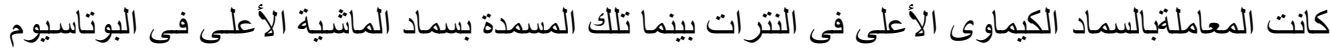

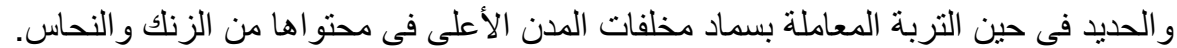

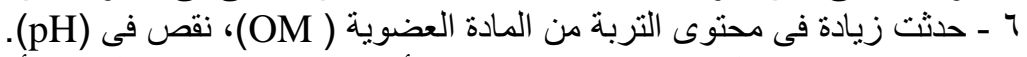

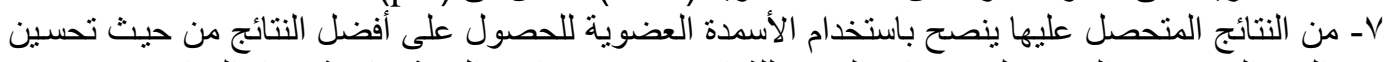
النمو الخضرى و المحصول وصفات الجودة للثمار وتحسين خو اص التربة خلنة خاصة سماد الدو اجن.

Fayoum J. Agric. Res. \& Dev., Vol.25, No.2, July, 2011 